\title{
Nab-paclitaxel plus gemcitabine as first-line palliative chemotherapy in a patient with metastatic pancreatic cancer with Eastern Cooperative Oncology Group performance status of 2
}

\author{
ANDRÉS J. MUÑOZ MARTÍN, PILAR GARCÍA ALFONSO, ANA B. RUPÉREZ and MIGUEL MARTÍN JIMÉNEZ
}

Medical Oncology Service, Hospital General Universitario Gregorio Marañón, Madrid 28007, Spain

Received March 4, 2015; Accepted March 24, 2016

DOI: $10.3892 / 01.2016 .4667$

\begin{abstract}
Metastatic pancreatic cancer (PC) has been associated with a considerably poor prognosis. Due to its toxicity, first-line combination chemotherapy is limited to patients with a good performance status (PS). Previously nab-paclitaxel plus gemcitabine has been demonstrated to improve the overall survival rate in patients with advanced pancreatic cancer with a good PS. The present study reports a case of a patient with metastatic PC with a poor PS (Eastern Cooperative Oncology Group 2) and a complex set of comorbidities treated with nab-paclitaxel plus gemcitabine as a first-line palliative therapy. Adjusted doses of nab-paclitaxel plus gemcitabine reached a favourable clinical, radiological and biochemical response in the present patient, which increased the quality of life for the patient. Eventually, the patient succumbed to acute cholangitis. Based on the results of the present study, nab-paclitaxel plus gemcitabine appears to be a favourable treatment as first-line palliative chemotherapy for patients with metastatic PC, comorbidities and poor PS.
\end{abstract}

\section{Introduction}

Pancreatic cancer is the fourth leading cause of cancer-associated mortality in western countries (1). Pancreatic cancer has the highest mortality rate of all major cancers, with a 5-year overall survival of 5-6\% across all disease stages. Survival rate has not improved substantially in the past 30 years. The number of novel cases is increasing, in the next decade an increase of $50 \%$ is expected (2). The prognosis of patients with metastatic pancreatic cancer (PC) is extremely poor (1). The modest results of current treatments (first/second-line) reveal the requirement for novel

Correspondence to: Dr Andrés J. Muñoz Martín, Medical Oncology Service, Hospital General Universitario Gregorio Marañón, C/Doctor Esquerdo 46, Madrid 28007, Spain

E-mail: andresmunmar@hotmail.com

Key words: pancreatic adenocarcinoma, nab-paclitaxel plus gemcitabine, palliative therapy, poor performance status, tolerance, CA19-9 therapeutic strategies (2). Surgery remains the only curative treatment for pancreatic cancer patients with a 5-year survival rate of $10-20 \%$ and patients diagnosed with metastatic disease median overall survival range from 6-11 months with systemic chemotherapy (3-6). The MPACT trial results revealed that nab-paclitaxel plus gemcitabine improved the overall survival (OS), progression-free survival (PFS) and response rate of patients compared with gemcitabine treatment alone (7). However, the associated toxicity limited its use in patients with a poor performance status (PS). Consequently these patients usually received single-agent chemotherapy.

The present study reports a case of a patient with metastatic PC with high comorbidity and poor PS [Eastern Cooperative Oncology Group (ECOG) 2] (8), who developed bone and lung metastases with elevated levels of gastrointestinal cancer-associated carbohydrate antigen 19-9 (CA19-9). Adjusted doses of nab-paclitaxel plus gemcitabine were administered as first-line palliative chemotherapy, through which a partial response was obtained, since the levels of CA19-9 were decreased (9) and bone pain was alleviated. Therefore, improving the quality of life of the patient.

\section{Case report}

A 61-year-old Caucasian female patient was diagnosed with stage I invasive lobular right breast carcinoma (pT1c-pN0-M0) (10) at Hospital General Universitario Gregorio Marañón (Madrid, Spain) in April 2006, where all subsequent treatment was provided. Estrogen receptor (ER) was $30 \%$, progesterone receptor (PR) status and c-erbB were negative and $\mathrm{Ki}-67$ was $60 \%$. Following a right radical mastectomy and homolateral axillary lymphadenectomy (0/14 tested nodes were positive), the patient received adjuvant chemotherapy ( 4 cycles, intravenously, cycles every 21 days; $60 \mathrm{mg} / \mathrm{m}^{2}$ adriamycin and $600 \mathrm{mg} / \mathrm{m}^{2}$ cyclophosphamide). Subsequently, anastrozole administration was initiated; however, due to intolerance, anastrozole was changed to letrozole 3 months later (oral administration for 5 years).

In June 2010, a nodule was detected in the pancreatic head/neck. A thoracoabdominal computed tomograph (CT) scan and echo-endoscopy revealed a focal hypodense lesion measuring $2.5 \mathrm{~cm}$ in diameter $(1.7 \times 1.5 \mathrm{~cm})$ at the junction of the body and neck of the pancreas, which was closely attached to the portal, superior-mesenteric and splenic vein confluence, 
A

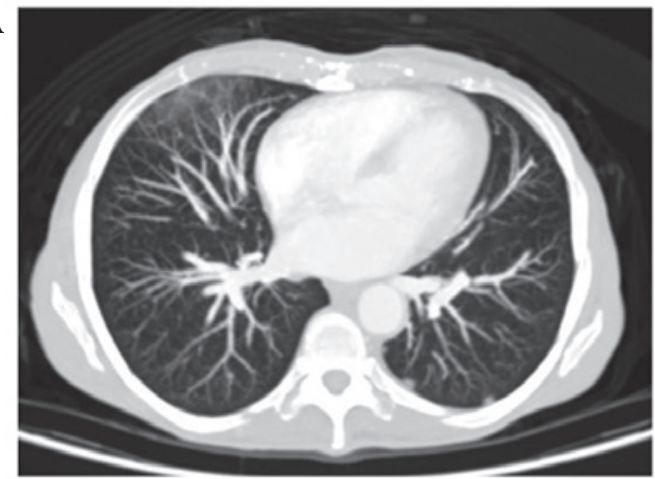

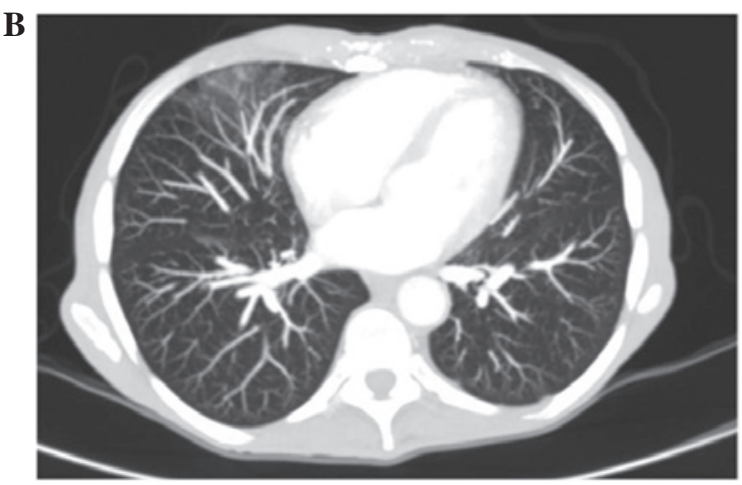

Figure 1. Positron emission tomography-computed tomography scan revealing the presence of subpleural pulmonary nodules in the posterior part of the left lower lobe (A) prior to and (B) following chemotherapy.

and small lymph nodes $(<1 \mathrm{~cm}$ in diameter) in the celiac, gastrohepatic and interaortocaval regions, without distant involvement. The CA19-9 levels were observed to be elevated (62 IU/ml; normal range, 2-37 IU/ml).

Pancreatic adenocarcinoma was diagnosed and confirmed by fine-needle aspiration puncture and positron emission tomography (PET)-CT (Biograph, Siemens, Germany), which demonstrated that there was increased metabolic activity in the tumor, without other macroscopic evidence of tumor activity.

Neoadjuvant chemoradiation was administered to the patient: Gemcitabine, $\left(1,000 \mathrm{mg} / \mathrm{m}^{2}\right.$; intravenous; days 1 , 8 and 15), followed by external beam radiotherapy (total dose, $50.4 \mathrm{~Gy} ; 5$ fractions/week of $1.8 \mathrm{~Gy} /$ fraction) with tegafur $(1,200 \mathrm{mg} /$ day orally, throughout radiotherapy treatment $)$ as the sensitizing agent. Non-Response Evaluation Criteria in Solid Tumors (non-RECIST) (11) partial response was confirmed by $\mathrm{CT}$, and a complete pancreaticoduodenectomy with splenectomy and vascular portal resection, including the splenic and superior-mesenteric veins with D2-lymphadenectomy, was performed. Intraoperative radiotherapy $(1,000 \mathrm{~Gy})$ was successfully delivered over the vascular risk area. The postoperative course of the patient was favourable with no significant findings.

Histopathology on the resected tumor confirmed an invasive ductal adenocarcinoma of the pancreas (ypT2-pN0-M0) with extensive stromal changes and hyalinization, and focal invasion of the peripancreatic tissue; peripancreatic lymph nodes and large-diameter vessels were not involved.

Subsequently, 3 cycles of gemcitabine adjuvant chemotherapy $\left(1,000 \mathrm{mg} / \mathrm{m}^{2}\right.$; intravenous; days 1,8 and 15$)$ was administered, following which the CA19-9 levels were observed to be normal $(15 \mathrm{IU} / \mathrm{ml})$. During treatment, dose delays occurred due to toxicity in the patient (grade 3 asthenia/neutropenia; grade 2 anemia), and subsequently the patient presented chemotherapy-induced post-pancreatectomy diabetes mellitus, exocrine pancreatic insufficiency, chronic post-radiation enteritis and recurrent acute cholangitis associated with chronic bile duct dilatation (thrombotic cholangiopathy).

In February 2011, post-chemotherapy PET-CT did not reveal any evidence of disease, but vascular thrombosis of portal and superior-mesenteric veins was observed. Therefore, the patient was administered with enoxaparin $(1 \mathrm{mg} / \mathrm{kg} / 12 \mathrm{~h}$, subcutaneously, for 6 months).

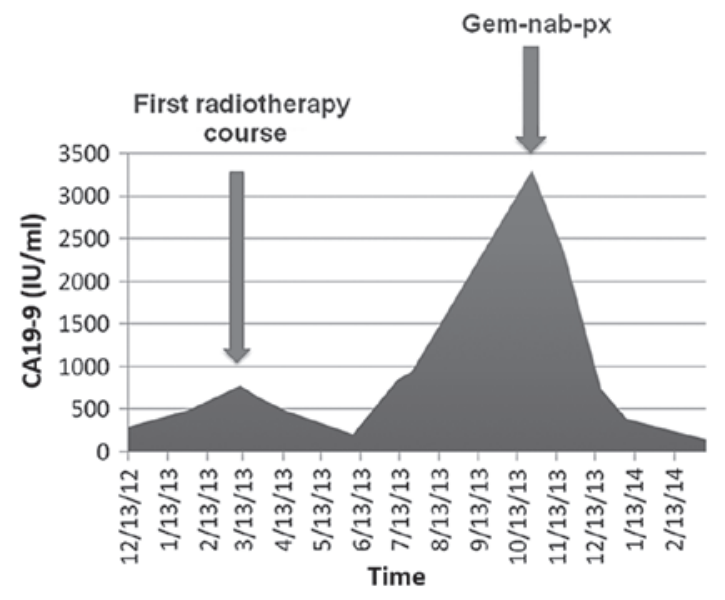

Figure 2. CA19-9 level variations pre- and post-chemotherapy with gem-nab-px. CA19-9, carbohydrate antigen 19-9; gem-nab-px, gemcitabine plus nab-paclitaxel.

In October 2012, a simple left mastectomy was performed for grade 2 microinvasive breast carcinoma ( $2 \mathrm{~mm}$ in size) without axillar involvement [ER/PR, negative; Ki-67, 60\%; human epidermal growth factor receptor 2 (HER2) gene amplification, positive; HER2/centromere enumerator probe 17 ratio, $>2.2$ (fluorescence in situ hybridization)]. No adjuvant therapy was administered.

In January 2013, the patient presented with intense lumbar pain, and chest/abdomen/pelvis CT revealed the presence of a sclerotic lesion involving the L5 vertebral body, without spinal canal invasion. The results of bone biopsy and PET-CT confirmed a diagnosis of sclerotic metastasis from moderately differentiated adenocarcinoma of pancreatic origin (cytokeratin (CK) 7, CA19-9, CK17 and CK19, positive; CK20, ER/PR and c-erb2, negative) without macroscopic evidence of active malignant disease in other locations. Chemotherapy, zoledronic acid (4 mg every 28 days, continuously) and radiotherapy at the L5 vertebra were proposed, but the patient refused chemotherapy. Therefore, zoledronic acid and external beam radiotherapy treatment was initiated to the lumbar spine in March-April 2013 (10x300 cGy, 10 fractions; total dose, $30 \mathrm{~Gy})$. Bone pain improved and CA19-9 levels decreased in June 2013 (650 and $215 \mathrm{IU} / \mathrm{ml}$, pre- and post-radiotherapy). However, 1 month later, intense lumbar pain in the L5 
reappeared and the CA19-9 levels increased to $858 \mathrm{IU} / \mathrm{ml}$; management with first-stage analgesia failed [paracetamol and non-steroidal anti-inflammatory drug (NSAID)]. The restaging evaluation did not reveal any evidence of malignancy in other locations. Chemotherapy and surgery were rejected by the patient; therefore, the patient received reirradiation over the same region ( $5 \times 400$ cGy, 5 fractions; total dose, $20 \mathrm{~Gy}$ ), which had a poor analgesic response.

In October 2103, during the follow-up period, the lumbar/sacral bone pain increased and novel abdominal pain appeared. PET-CT demonstrated malignant activity in the abdominopelvic lymph nodes, several bone lesions at T11, T12 and L5 vertebrae and bilateral lung nodules (Fig. 1A); a questionable secondary involvement of the left adrenal gland was also detected. Reirradiation was dismissed and the patient underwent chemotherapy. The patient was not eligible for FOLFIRINOX and capecitabine due to her ECOG PS of 2, history of chronic diarrhea and post-pancreatectomy malabsorption. As gemcitabine had been previously administered, weight-adjusted gemcitabine $\left(400 \mathrm{mg} / \mathrm{m}^{2}\right.$; intravenously; days $1,8$ and 15$)$ plus nab-paclitaxel $\left(50 \mathrm{mg} / \mathrm{m}^{2}\right.$; intravenously; days 1,8 and 15) was administered in November 2013 as first-line palliative chemotherapy every 28 days.

Patient characteristics prior to treatment were as follows: Weight, $46 \mathrm{~kg}$; height, $164 \mathrm{~cm}$; body mass index, 17.1; ECOG PS, 2; CA19-9, $3306 \mathrm{IU} / \mathrm{ml}$; and analgesia (oral oxycodone $40 \mathrm{mg} / 12 \mathrm{~h}$ ). Following the second chemotherapy cycle in January 2014, chest/abdomen/pelvis CT revealed stabilization of bone lesions and abdominal lymph nodes and no pulmonary metastasis (Fig. 1B). In addition, treatment lead to a progressive CA19-9 reduction (Fig. 2) and bone and abdominal pain improvement (analgesic response in lumbosacral spine). The CA19-9 levels of the patient decreased markedly (285 IU/ml), analgesia was gradually reduced to $5 \mathrm{mg} / 12 \mathrm{~h}$, and an improvement in nocturnal rest and slight body weight increase were noted. Overall, the tolerance was acceptable. Toxicities included grade 2 anemia/lymphocytopenia/asthenia, non-neutropenic fever associated with cholangitis and grade 1 diarrhea, vomiting, hepatotoxicity and lower-limb edema.

Chemotherapy was continued at the same dose, due to the good maintained response, including the excellent analgesic response and a decreased in ECOG PS to 1. Eventually, the patient succumbed to acute cholangitis in April 2014.

\section{Discussion}

The present case report demonstrates the efficacy and safety of combination therapy of nab-paclitaxel plus gemcitabine in a patient with stage IV PC with an ECOG PS of 2 and several comorbidities, including breast carcinoma and bone (L5 vertebral body) and lung metastases.

Isolated bone metastasis in PC is uncommon (12); therefore, in the present study, the malignancy and origin of the bone lesion was confirmed by biopsy prior to treatment (pancreatic adenocarcinoma instead of breast cancer). For this advanced PC, radical surgery at the L5 was dismissed, due to a lack of clear benefit. With regard to chemotherapy treatment options, 4 different regimens of first-line chemotherapy that have shown an OS benefit in advanced PC have been reported: Gemcitabine (13), gemcitabine plus erlotinib (14), FOLFIRINOX (15) and nab-paclitaxel plus gemcitabine (7).

For the present patient, who had previously received gemcitabine monotherapy (adjuvant and neo-adjuvant), oral erlotinib and FOLFIRINOX were not considered due to malabsorption, since the patient had diarrhea due to exocrine pancreatic insufficiency and chronic post-radiation enteritis, and the unfavourable toxic profile of these two combinations $(14,15)$. Nab-paclitaxel plus gemcitabine combination treatment has demonstrated efficacy in patients with PC. In the MPACT trial (7) [Karnofsky PS $\geq 70$, equivalent to ECOG PS $\leq 2$ (16)] nab-paclitaxel plus gemcitabine combination improved the OS and PFS of patients compared with patients treated with gemcitabine alone. Another clinical case reported that in a patient with ECOG PS of 3 nab-paclitaxel plus gemcitabine combination treatment was also efficient (17).

In the MPACT trial (7), a $\geq 90 \%$ reduction in the CA19-9 levels was observed primarily in patients treated with nab-paclitaxel plus gemcitabine versus patients treated with gemcitabine alone (31 vs. $14 \%$; $\mathrm{P}<0.001)$. In addition, the nab-paclitaxel plus gemcitabine combination treatment was associated with a longer median survival time (13.5 vs. 8.2 months; $\geq 90$ vs. $<90 \%$ CA19-9 decrease). In the present patient a $\geq$ CA19-9 reduction was also observed.

An ongoing Spanish randomized phase I study (FRAGANCE) is evaluating the efficacy of nab-paclitaxel plus gemcitabine treatment in patients with PC (ECOG PS 2) (10). The promising results of phase I demonstrated the feasibility of this combination (standard-dose/low-dose regimen) in these patients, which is supported by the present results.

The present patient received nab-paclitaxel plus gemcitabine every 28 days. The excellent clinical response and early CA19-9 reduction rendered a dose increase unnecessary. Pain levels of the patient could be markedly controlled by reducing the oxycodone dose. The toxicity observed was consistent with that observed in the MPACT (7) and FRAGANCE (phase-I) (18) trials.

In conclusion, the findings of the present study demonstrated that nab-paclitaxel plus gemcitabine combination treatment is a favorable treatment option with acceptable tolerance for patients with metastatic PC and other comorbidities.

\section{References}

1. American Cancer Society: Cancer Facts \& Figures. American Cancer Society, Atlanta, GA, 2014.

2. Malvezzi M, Bertuccio P, Levi F, La Vecchia C and Negri E: European cancer mortality predictions for the year 2013. Ann Oncol 24: 792-800, 2013.

3. Neoptolemos JP, Dunn JA, Stocken D, Almond J, Link K, Beger H, Bassi C, Falconi M, Pederzoli P, Dervenis C, et al: Adjuvant chemoradiotherapy and chemotherapy in resectable pancreatic cancer: A randomised controlled trial. Lancet 358: 1576-1585, 2001

4. Vincent A, Herman J, Schulick R, Hruban RH and Goggins M: Pancreatic cancer. Lancet 378: 607-620, 2011.

5. Adsay NV, Basturk O, Cheng JD and Andea AA: Ductal neoplasia of the pancreas: nosologic, clinicopathologic, and biologic aspects. Semin Radiat Oncol 15: 254-264, 2005.

6. Bilimoria KY, Bentrem DJ, Ko CY, Stewart AK, Winchester DP and Talamonti MS: National failure to operate on early stage pancreatic cancer. Ann Surg 246: 173-180,2007.

7. Von Hoff DD, Ervin T, Arena FP, et al: Increased survival in pancreatic cancer with nab-paclitaxel plus gemcitabine. N Engl J Med 369: 1691-1703, 2013. 
8. Oken MM, Creech RH, Tormey DC, Horton J, Davis TE, McFadden ET, et al: Toxicity and response criteria of the Eastern Cooperative Oncology Group. Am J Clin Oncol 5: 649-655, 1982

9. Ferrone CR, Finkelstein DM, Thayer SP, Muzikansky A, Fernandez-delCastillo $\mathrm{C}$ and Warshaw AL: Perioperative CA19-9 levels can predict stage and survival in patients with resectable pancreatic adenocarcinoma. J Clin Oncol 24 2897-2902, 2006.

10. Sobin LH, Gospodarowicz MK and Wittekind C (Eds): TNM Classification of Malignant Tumours, 7th edition. Wiley-Blackwell, Hoboken, NJ, USA. pp 3241-3244, 2009.

11. Therasse P, Arbuck SG, Eisenhauer EA, Wanders J, Kaplan RS, Rubinstein L, et al: New guidelines to evaluate the response to treatment in solid tumors. European Organization for Research and Treatment of Cancer, National Cancer Institute of the United States, National Cancer Institute of Canada. J Natl Cancer Inst 92: 205-216, 2009.

12. Borad MJ, Saadati H, Lakshmipathy A, Campbell E, Hopper P, Jameson G, Von Hoff DD and Saif MW: Skeletal metastases in pancreatic cancer: A retrospective study and review of the literature. Yale J Biol Med 82: 1-6, 2009.

13. Burris HA 3rd, Moore MJ, Andersen J, Green MR, Rothenberg ML, Modiano MR, et al: Improvements in survival and clinical benefit with gencitabine as first-line therapy for patients with advanced pancreas cancer: A randomized trial. J Clin Oncol 15: 2403-2413, 2007.
14. Moore MJ, Goldstein D, Hamm J, Figer A, Hecht JR, Gallinger S, Au HJ, Murawa P, Walde D, Wolff RA, et al: Erlotinib plus gemcitabine compared with gemcitabine alone in patients with advanced pancreatic cancer: A phase III trial of the National Cancer Institute of Canada Clinical Trials Group. J Clin Oncol 25: 1960-1966, 2007.

15. Conroy T, Desseigne F, Ychou M, Bouché O, Guimbaud R, Bécouarn Y, Adenis A, Raoul JL, Gourgou-Bourgade S, de la Fouchardière $\mathrm{C}$, et al: FOLFIRINOX versus gemcitabine for metastatic pancreatic cancer. N Engl J Med 364: 1817-1825, 2011.

16. Buccheri G, Ferrigno D and Tamburini M: Karnofsky and ECOG performance status scoring in lung cancer: A prospective, longitudinal study of 536 patients from a single institution. Eur J Cancer 32A: 1135-1141, 1996.

17. Shakir AR: A Near-Complete response to treatment with gemcitabine plus nab $\left({ }^{\circledR}\right)$-Paclitaxel in a patient with metastatic pancreatic cancer and poor performance status: A case report. Case Rep Oncol 7: 711-717, 2014.

18. Guillen-Ponce C, López R, Macarulla T, Rivera F, Cubillo A Carrato A, et al: A phase I/II trial to evaluate the efficacy and safety of nab-paclitaxel in combination with gemcitabine for the treatment of frail patients with advanced or metastatic pancreatic cancer: Safety results of the Phase I trial. Ann Oncol 25 (suppl 4): iv238, 2014 